\title{
A new detection scheme for van der Waals heterostructures, imaging individual fullerenes between graphene sheets, and controlling the vacuum in scanning transmission electron microscopy
}

\author{
G. Argentero ${ }^{1}$, K. Mustonen ${ }^{1}$, R. Mirzayev ${ }^{1}$, A. Mittelberger ${ }^{1}$, T. Susi ${ }^{1}$, G.T. Leuthner ${ }^{1}$, Y. Cao ${ }^{2}$, M.R.A. \\ Monazam $^{1}$, T.J. Pennycook ${ }^{1}$, C. Mangler ${ }^{1}$, C. Kramberger ${ }^{1}$, A.K. Geim², J. Kotakoski ${ }^{1}$, J. C. Meyer ${ }^{1}$ \\ 1. University of Vienna, Faculty of Physics, 1090 Vienna, Austria \\ 2. Centre for Mesoscience and Nanotechnology and School of Physics and Astronomy, University of \\ Manchester, Manchester, M13 9PL, United Kingdom
}

This presentation contains three topics that are of current interest in particular for low-dimensional materials. First, I will describe a new detection scheme that allows us to unveil the deformations in a graphene-hBN heterostructure [1], where the two layers have only a very small lattice mismatch. We use a pixelated detector for recording the scattered intensity in the angular range of the annular darkfield detector. The asymmetry in the scattered intensity turns out to be very sensitive to the local projected displacement between the two layers (Fig. 1). By comparing the experimental signal with simulations for different atomistic models, we establish the 3D structure of the sample [1].

The second topic are van der Waals heterostructures of mixed dimensionality, in our case a sandwich structure made of two graphene layers that encapsulate one or a few layers of fullerenes. By using the graphene sandwich, clean and well defined layers of the $\mathrm{C}_{60}$ molecule can be observed. We studied the dynamics of individual molecules in monolayers of fullerenes between the graphene sheets. In particular we can distinguish between freely rotating fullerenes, and fullerenes that form bonds (due to damage) in such a way that their rotation is hindered [2].

The third topic concerns the interaction of 2D materials, especially graphene, with reactive gases in presence of an electron beam. Since low-dimensional materials are only one or few atomic layers thick, practically all atoms are exposed on the surface and therefore always in interaction with the environment. Our Nion UltraSTEM 100 was modified to reach pressures down to $\sim 3 \cdot 10^{-10}$ mbar in the sample area [3], and in particular, to allow sample transfer in vacuum from an external ultra-high vacuum (UHV) preparation chamber (Fig. 3a). The sample can also be exposed to various gases up to a pressure of $10^{-6}$ mbar during imaging. Fig. 3b shows a graphene edge under UHV conditions, where we find predominantly armchair edges that are very stable under $60 \mathrm{kV}$ electron irradiation at room temperature (RT). This behavior is in agreement with theoretical predictions, but in contrast to previous experimental findings which reported predominantly zigzag edges for holes that formed under electron irradiation at RT (e.g. [4]). However, in presence of oxygen $\left(10^{-6} \mathrm{mbar}\right)$ and electron irradiation, the armchair edge is decomposed more rapidly than the zigzag edge, and we also observe predominantly the zigzag configuration (Fig. 3c).

[1] G Argentero et al., Nano letters (in press, 2017), DOI: 10.1021/acs.nanolett.6b04360

[2] R Mirzayev et al. (submitted, 2017)

[3] M Hotz et al., Microscopy and Microanalysis, 22(S3), (2016) p. 34.

[4] CO Girit et al., Science 323 (2009) p. 1705 
[5] The authors acknowledge funding from the European Research Council (ERC) projects PICOMAT and ARTIMATTER, the Austrian Science Fund (FWF) projects P25721-N20 and P 28322-N36, and the Wiener Wissenschafts- Forschungs- und Technologiefonds (WWTF) via project MA14-009.
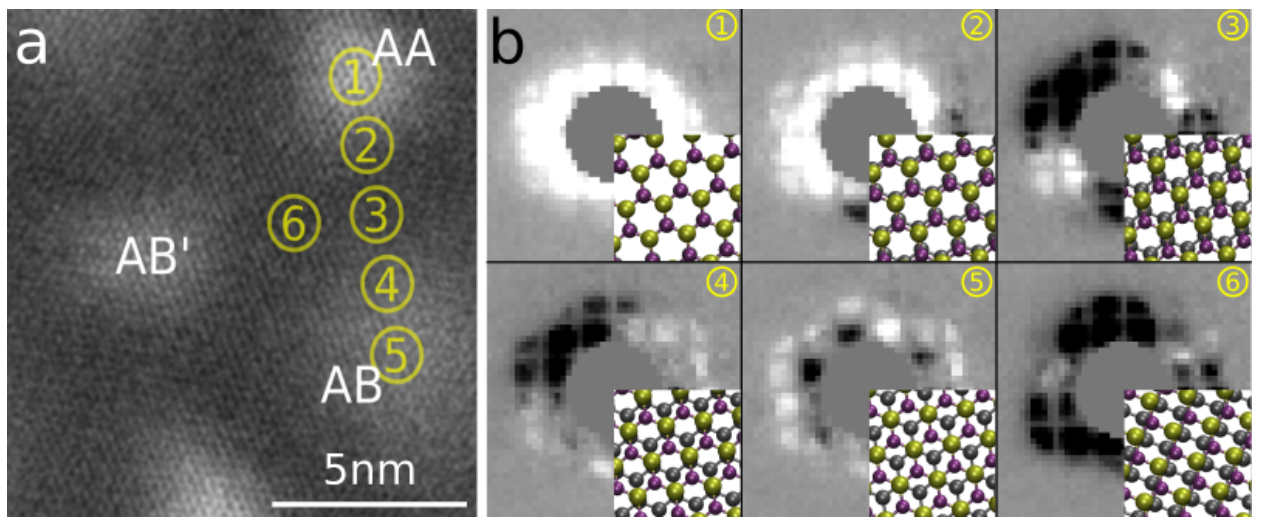

Figure 1. (a) Medium-angle annular dark field (MAADF) image of a graphene-hBN heterostructure. (b) Images of the MAADF scattering intensity distribution from indicated positions in the map. Insets illustrate the local stacking of the two layers that is associated to these points.

a
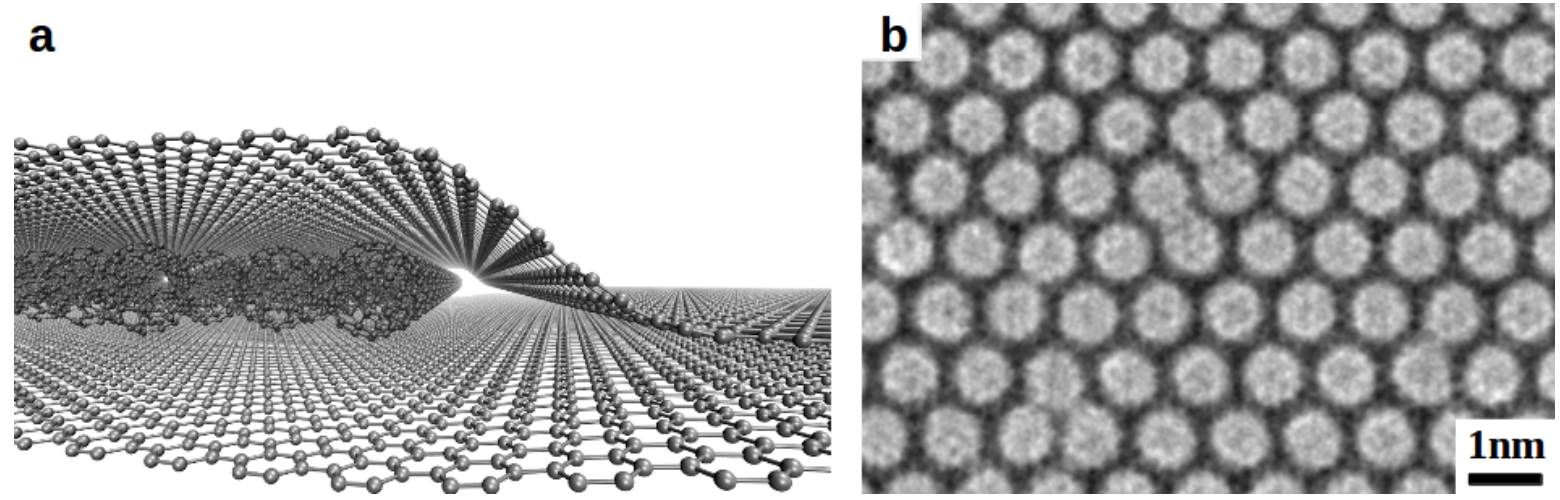

Figure 2. (a) Illustration of a graphene-fullerene sandwich. (b) STEM image of a $\mathrm{C}_{60}$ monolayer between two graphene sheets.
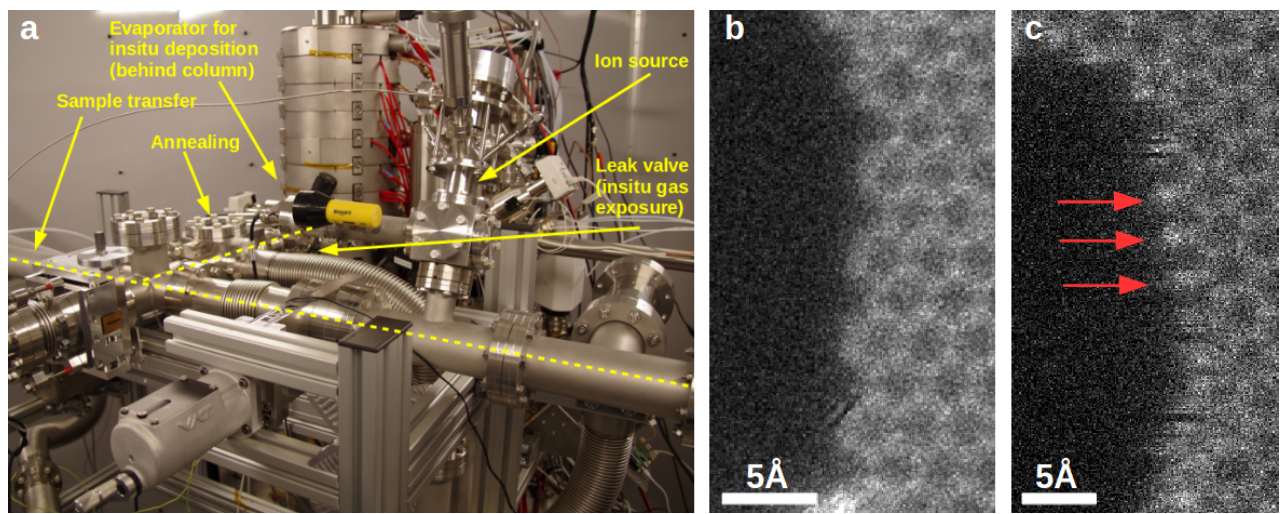

Figure 3. (a) Photo of the UltraSTEM with part of the external UHV system. (b) Graphene edge in UHV is found predominantly in the armchair configuration. (c) Graphene edge imaged in $10^{-6}$ mbar of oxygen displaying mostly the zigzag orientation. The red arrows indicate heavier atoms at the edge, identified as oxygen atoms. 